\title{
Patulin induces pro-survival functions via autophagy inhibition and p62 accumulation
}

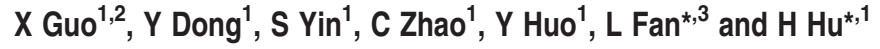

Patulin (PAT) is one of the most common mycotoxins found in moldy fruits. Skin contact is one of the most likely exposure routes of PAT. Investigation of dermal toxicity of PAT is clearly needed and has been highlighted by WHO. In the present study, using human keratinocyte $\mathrm{HaCaT}$ cells as a model, we found that treatment with PAT caused an increased autophagosome accumulation. Measurements of autophagic flux demonstrated that the accumulation of autophagosomes by PAT was not directly due to enhanced autophagosome formation but due to inhibition of autophagosome degradation. Reductions in the activities of the lysosomal enzymes cathepsin B and cathepsin D by PAT might contribute to this inhibitory effect. Consistent with this, inhibition of autophagosome degradation by PAT resulted in accumulation of p62 that functioned as a pro-survival signal. The pro-survival function of $\mathrm{p} 62$ was found to be attributed to reactive oxygen species-mediated cytoprotective endoplasmic reticulum (ER) stress response. ER stress exerted cytoprotective effect via extracellular signal-regulated kinase1/2dependent B-cell CLL/lymphoma 2-associated agonist of cell death inhibitory phosphorylation. Given the critical role of autophagy and its substrate p62 in carcinogenesis, our findings may have important implications in PAT-induced skin carcinogenesis.

Cell Death and Disease (2013) 4, e822; doi:10.1038/cddis.2013.349; published online 3 October 2013

Subject Category: Cancer

Autophagy is the process by which cells break down their own long-lived proteins and damaged components, such as mitochondria. ${ }^{1}$ Growing evidence has demonstrated that autophagy has important and paradoxical roles in carcinogenesis. Several studies have shown that autophagy functions as tumor suppressor and autophagy deficiency indeed induces an increased frequency of spontaneous cancer of multiple organ sites, including lung, liver and lymphocytes. ${ }^{2-6}$ In contrast, several other studies argue that autophagy could support tumor progression and survival. ${ }^{7,8}$ p62 (also known as sequestosome-1), a molecular adapter protein between the autophagic machinery and its substrates, has been suggested to have a critical role in autophagy-regulated carcinogenesis. ${ }^{9,10}$

Patulin (PAT), a mycotoxin produced by a variety of molds, particularly Aspergillus and Penicillium, is frequently found in moldy fruits and their derivative products. ${ }^{11}$ Skin contact with these fruits is likely to be the chief route of exposure for PAT. Therefore, it is clearly needed to evaluate dermal toxicity of PAT. ${ }^{12}$ It has been shown that single topical applications of PAT followed by twice weekly applications of TPA caused tumor formation. ${ }^{13}$ To further investigate these findings, we found that PAT caused the inhibition of autophagosome degradation that was associated with reducing the activities of Iysosomal enzymes cathepsin B (CTSB) and cathepsin D (CTSD) in human keratinocytes. Autophagy inhibition led to accumulation of $\mathrm{p62}$, followed by increased reactive oxygen species (ROS) generation that triggered cytoprotective endoplasmic reticulum (ER) stress response. This resulted in BCL2-associated agonist of cell death (BAD) inhibitory phosphorylation by extracellular signal-regulated kinase (ERK)1/2. Our findings uncovered a novel mechanism involved in the pro-survival function of p62. Disruption of apoptosis by inhibitory phosphorylation of BAD might contribute to PAT-induced skin carcinogenesis.

\section{Results}

PAT inhibits autophagosome degradation in HaCaT and 293T cells. To determine whether PAT can regulate autophagy induction, human keratinocyte $\mathrm{HaCaT}$ cells were treated with PAT at concentrations of $3-7 \mu \mathrm{M}$ for $24 \mathrm{~h}$ and

\footnotetext{
${ }^{1}$ Department of Nutrition and Health, College of Food Science and Nutritional Engineering, China Agricultural University, Beijing, China; ${ }^{2}$ Laboratory of Food Engineering and Nutrition Research, Yellow Sea Fisheries Research Institute, Chinese Academy of Fishery Science, Qingdao, China and ${ }^{3}$ College of Veterinary Medicine, China Agricultural University, Beijing, China

*Corresponding authors: $\mathrm{H} \mathrm{Hu}$, Department of Nutrition and Health, College of Food Science and Nutritional Engineering, China Agricultural University, No 17 Qinghua East Road, Haidian District, Beijing 100083, China. Tel: + 86106273 8653; Fax: + 86106273 8653; E-mail: hongbo @ cau.edu.cn

or L Fan, College of Veterinary Medicine, China Agricultural University, No 2 Yunamingyuan West Road, Haidian District, Beijing 100193, China. Tel: + 86106273 3322; Fax: + 86106273 3322; E-mail: flh@ cau.edu.cn

Keywords: patulin; autophagy; p62; UPR; bad phosphorylation

Abbreviations: IRE $1 \alpha$, inositol requiring ER-to-nucleus signal kinase- $1 \alpha$; PERK, protein kinase RNA-like endoplasmic reticulum kinase; elF2 $\alpha$, eukaryotic initiation factor 2 $\alpha$; GRP78, glucose-regulated protein, 78 kDa; MCL1, myeloid cell leukemia sequence 1 (BCL2-related); BCL2, B-cell CLL/lymphoma 2; Bcl-xL, B-cell lymphoma extra-large; ERK, extracellular signal-regulated kinase; BAD, BCL2-associated agonist of cell death; LC3, microtubule-associated protein 1 light chain 3; PARP1, poly (ADP-ribose) polymerase 1; p62, SQSTM1, sequestosome-1; ATG5, autophagy-related 5; CTSB, cathepsin B; CTSD, cathepsin D; CHX, cycloheximide; 3-MA, 3-methyladenine; BAF, bafilomycin A1; NAC, N-acetyl-I-cysteine; ER, endoplasmic reticulum; UPR, unfolded protein response; PAT, patulin

Received 06.5.13; revised 07.8.13; accepted 27.8.13; Edited by GM Fimia
} 
then a lipidated form of microtubule-associated protein 1 light chain 3 (LC3), LC3-II, an autophagic marker, was analyzed by western blotting. As shown in Figure 1a, treatment with PAT led to a concentration-dependent increase in LC3-II level. Furthermore, LC3 lipidation by PAT was further verified by immunofluorescence staining to analyze distribution patterns of LC3. As shown in Figure 1b, a diffuse localization of LC3 fluorescence was observed in control cells, whereas a punctuated pattern of LC3 fluorescence was detected in PAT-treated cells. Quantitative analysis of LC3 punctuated cells showed that the number of cells with LC3 puncta in PAT-treated cells was significantly increased compared with the untreated control (Figure 1c). To confirm PAT-induced LC3 lipidation is indeed due to autophagosome formation, we

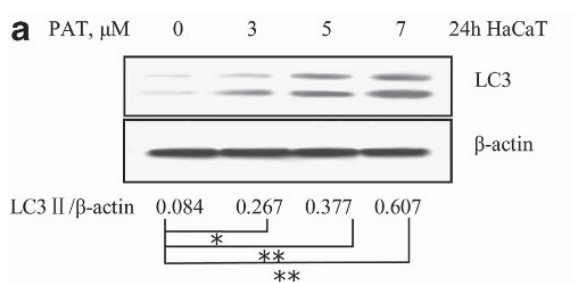

b

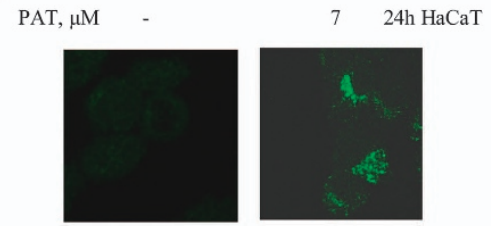

C

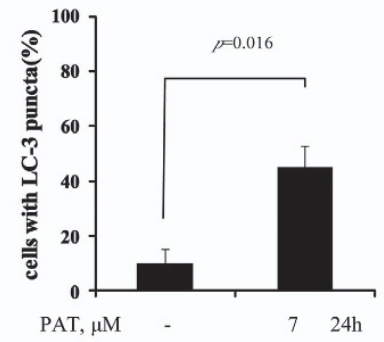

e

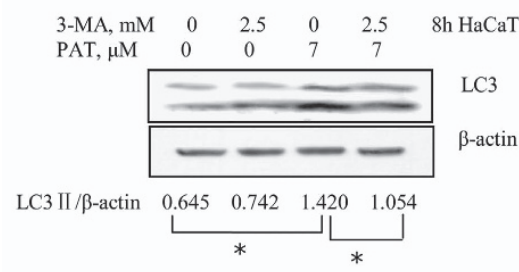

d CONsi,nM $60 \quad-\quad 60 \quad-\quad 24 \mathrm{~h}$ ATG5si,nM - $60 \quad-60$ PAT, $\mu \mathrm{M} \quad-\quad-\quad 7 \quad 4$

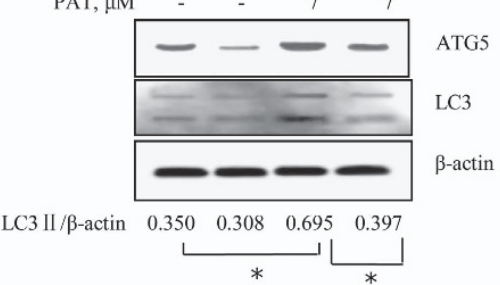

f

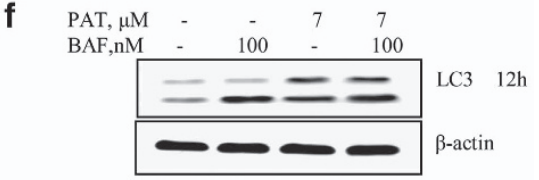

LC3 II / $\beta$-actin $\quad 0.279 \quad 0.645 \quad 0.551 \quad 0.648$

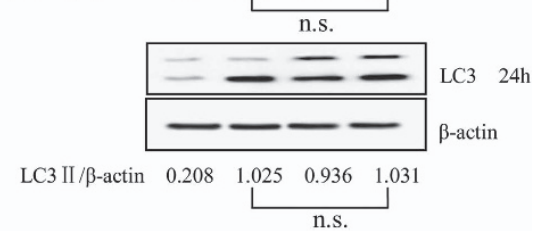

g

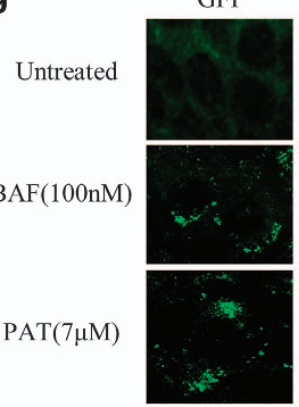

RFP

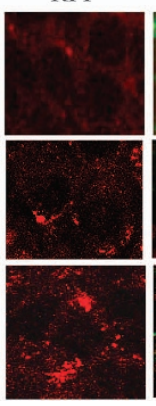

Merge

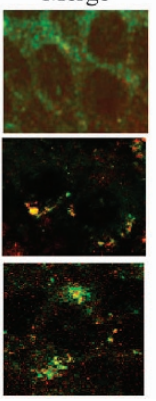

Figure 1 PAT induces autophagy inhibition in HaCaT cells. (a) PAT caused an increased LC3-I to LC3-II conversion in HaCaT cells analyzed by western blotting (24 h). (b) A punctuated distribution of LC3 induced by PAT in HaCaT cells detected by immunofluorescence staining (24 h). (c) Quantitative analysis of LC3 punctuated cells in PAT-treated and -untreated HaCaT cells. (d) Effects of ATG5 knockdown on the levels of ATG5 and LC3 in HaCaT cells. The cells were transfected with $60 \mathrm{nmol} / \mathrm{l}$ of ATG5 siRNA using siPORT NeoFX transfection agent. At $24 \mathrm{~h}$ post transfection, the cells were treated with $7 \mu \mathrm{M}$ PAT for $24 \mathrm{~h}$, then protein levels were detected by western blotting. (e) Effects of autophagy inhibition by 3-MA on the levels of LC3 by PAT treatment in HaCaT cells. The cells were pretreated with 3-MA for $1 \mathrm{~h}$ and further treated with $7 \mu \mathrm{M} \mathrm{PAT}$ for $8 \mathrm{~h}$ and western blotting was used to analyze LC3. (f) Inhibition of autophagosome degradation was involved in PAT-induced autophagosome in HaCaT cells. The cells were treated with $7 \mu \mathrm{M}$ PAT for 12 and $24 \mathrm{~h}$ in the presence or absence of $100 \mathrm{nM} \mathrm{BAF}$ (added $2 \mathrm{~h}$ before cell harvest) and then LC3 was analyzed by western blotting. (g) Measurement of autophagic flux by immunofluorescence using mRFP-GFP-LC3 reporter. HaCaT cells were transfected with plasmid expressing mRFP-GFP-LC3 (Addgene, Cambridge, MA, USA) using Lipofectamine 2000 (Invitrogen, Carlsbad, CA, USA). Twenty-four hours after transfection, the cells were treated BAF (100 nM) or PAT $(7 \mu \mathrm{M})$ and the fluorescence was observed under confocal microscope. $n=3,{ }^{*} P<0.05,{ }^{* *} P<0.01$, n.s., no significant difference 
tested effects of autophagy inhibition by either knockdown of autophagy-related 5 (ATG5) or a chemical inhibitor 3-methyladenine (3-MA) ${ }^{14}$ on PAT-induced LC3-I/LC3-II conversion. The data are shown in Figures $1 \mathrm{~d}$ and $\mathrm{e}$. Inhibition of autophagy by ATG5 siRNA or 3-MA resulted in a significant attenuation of PAT-induced LC3-I/LC3-II conversion in HaCaT cells. To investigate whether the elevated levels of LC3 lipidation induced by PAT are due to increased formation of autophagosome or due to decreased degradation, we measured autophagic flux. As shown in Figure 1f, treatment with bafilomycin $\mathrm{A} 1$ (BAF), an inhibitor of autophagosome degradation, ${ }^{15}$ was expected to increase the accumulation of LC3-II, whereas PAT treatment for 12 or $24 \mathrm{~h}$ failed to further increase LC3-II level in the presence of BAF, suggesting that the inhibition of autophagosome degradation might contribute to PAT-induced accumulation of autophagosome in $\mathrm{HaCaT}$ cells. To further confirm this, we measured the flux by immunofluorescence using the mRFP-GFP-LC3 reporter. ${ }^{16}$ As shown in Figure 1g, the GFP and mRFP fluorescence were almost completely colocalized, further supporting that autophagosome degradation inhibition contributed to the accumulation of autophagosome by PAT. Similar results were also found in human embryonic kidney 293T cells (kidney is one of the key toxicological targets of PAT) (Supplementary Figures S1A-D online), suggesting general application of PAT-induced autophagy inhibition in its targeting cells.

Inhibition of autophagosome degradation-mediated induction of p62 protects PAT-induced apoptosis in HaCaT cells. Inhibition of autophagosome degradation by PAT was expected to increase p62 protein level. We analyzed effects of PAT on p62 protein level by western blotting. As shown in Figure 2a, PAT treatment caused a concentration-dependent induction of p62. To confirm p62 accumulation is indeed associated with inhibition of autophagosomes degradation, we examined influences of BAF on p62 induction by PAT. As shown in Figure $2 \mathrm{~b}$, under the condition that autophagosome degradation was blocked by its inhibitor, no further increase of $\mathrm{p} 62$ by PAT was observed, suggesting that the degradation mechanism was indeed involved in PAT-induced p62 induction. To further verify this notion, we assessed effects of cycloheximide (CHX), a protein synthesis inhibitor, on PAT-induced p62 accumulation. As shown in Figure 2c, inhibition of protein synthesis by $\mathrm{CHX}$ was expected to decrease p62 level. Under such condition, PAT treatment was still capable of inducing p62 accumulation, further supporting that the inhibition of protein turnover contributed to p62 induction by PAT. It has been shown that p62 functions either as pro-survival or proapoptotic signaling depending on the specific stimulus and possibly other conditions. ${ }^{17,18}$ To determine the functional role of p62 in response to PAT exposure, we evaluated influences of p62 knockdown on PAT-induced apoptosis by Annexin $\mathrm{V}$ staining and a Cell Death Detection ELISA kit. As shown in Figures $2 \mathrm{~d}$ and e, when $\mathrm{p} 62$ was silenced by RNA interference (RNAi), poly (ADP-ribose) polymerase 1 (PARP1) cleavage and apoptosis induced by PAT were significantly increased compared with PAT/control siRNA treatment, suggesting that induction of p62 acts as pro-survival signaling in response to PAT exposure.
PAT induces p62-mediated cytoprotective ER stress. It has been shown that autophagy inhibition can trigger ER stress in certain model systems. ${ }^{10,19}$ We therefore hypothesized that autophagy inhibition-mediated p62 induction by PAT might exert its pro-survival function via the induction of cytoprotective ER stress. HaCaT cells were treated with various concentrations of PAT for $24 \mathrm{~h}$ and then the changes of the key ER stress markers were analyzed by western blotting. As shown in Figure 3a, PAT treatment in the concentration range of 3-7 $\mu \mathrm{M}$ significantly increased protein abundance of ER chaperone glucose-regulated protein, $78 \mathrm{kDa}$ (GRP78), phosphorylation of the ER resident kinase protein kinase RNA-like endoplasmic reticulum kinase (PERK) and inositol requiring ER-to-nucleus signal kinase- $1 \alpha$ (IRE $1 \alpha$ ), eukaryotic initiation factor $2 \alpha$ (elF2 $\alpha$ ), a downstream target of PERK, suggesting that unfolded protein response (UPR) was activated in response to PAT exposure. To determine the functional role of PAT-induced UPR, we tested effects of knockdown of IRE $1 \alpha$ or PERK on PAT-induced apoptosis analyzed by a Cell Death Detection ELISA kit and Annexin V staining. As shown in Figure $3 b$, the protein levels of IRE $1 \alpha$ or PERK were dramatically decreased by their siRNAs. Under such condition, PAT-induced apoptosis was significantly increased compared with PAT/control siRNA treatment, indicating both IRE $1 \alpha$ and PERK had a cytoprotective role in response to PAT exposure. We next asked whether activation of UPR induced by PAT was attributed to p62 induction. p62 was silenced by RNAi and the changes of ER stress markers were measured and analyzed by western blotting. As shown in Figure 3c, when p62 was inhibited by its siRNA, phosphorylation of IRE $1 \alpha$ and elF $2 \alpha$ induced by PAT was significantly attenuated, supporting our hypothesis that p62 induction by PAT triggered cytoprotective ER stress response.

Inhibition of autophagosome degradation by BAF induced p62-mediated ER stress. To further confirm the role of p62 in autophagy inhibition-mediated UPR activation, we next investigated the function of p62 in autophagosome degradation inhibitor BAF-induced activation of UPR. HaCaT cells were treated with various concentrations of BAF for $12 \mathrm{~h}$ and then the changes of the key ER stress markers were analyzed by western blotting. As we expected, BAF treatment caused a concentration-dependent accumulation of LC3-II and p62 that was due to the inhibition of autophagosome degradation. These events were followed by a significantly increased phosphorylation of IRE $1 \alpha$ and elF2 $\alpha$, the key markers of ER stress, suggesting autophagy inhibition by BAF indeed caused ER stress response. We then examined effects of p62 inhibition by RNAi on BAFinduced UPR activation. As shown in Figure $4 b$, under the condition that p62 was silenced, phosphorylation of IRE1 $\alpha$ and elF $2 \alpha$ induced by BAF was dramatically suppressed, further supporting the critical role of p62 in autophagy inhibition-mediated ER stress response.

ROS is involved in PAT-induced p62-mediated ER stress. It has been shown that $\mathrm{p} 62$ accumulation can cause an increased ROS generation. ${ }^{10,20}$ We then questioned 

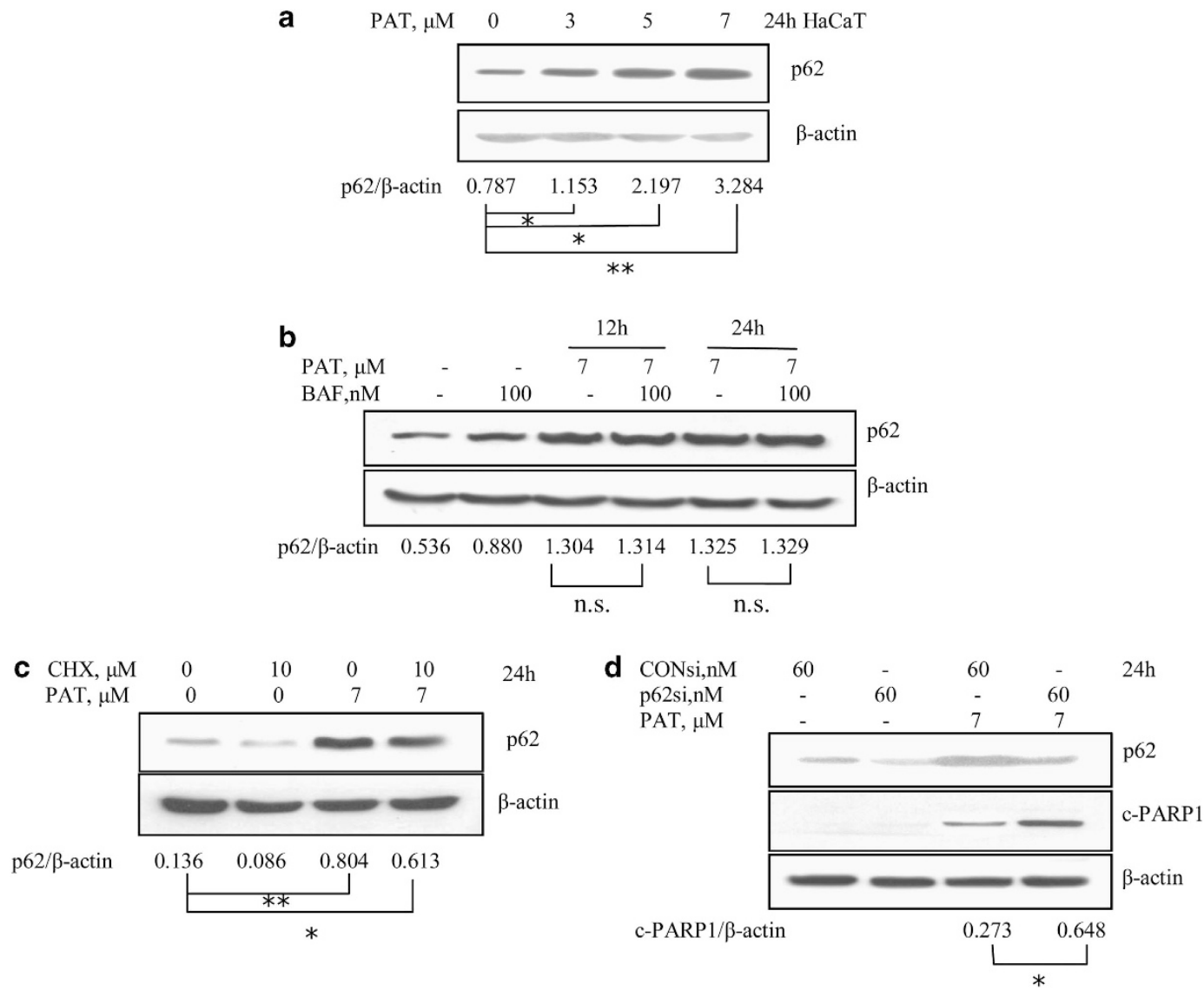

e
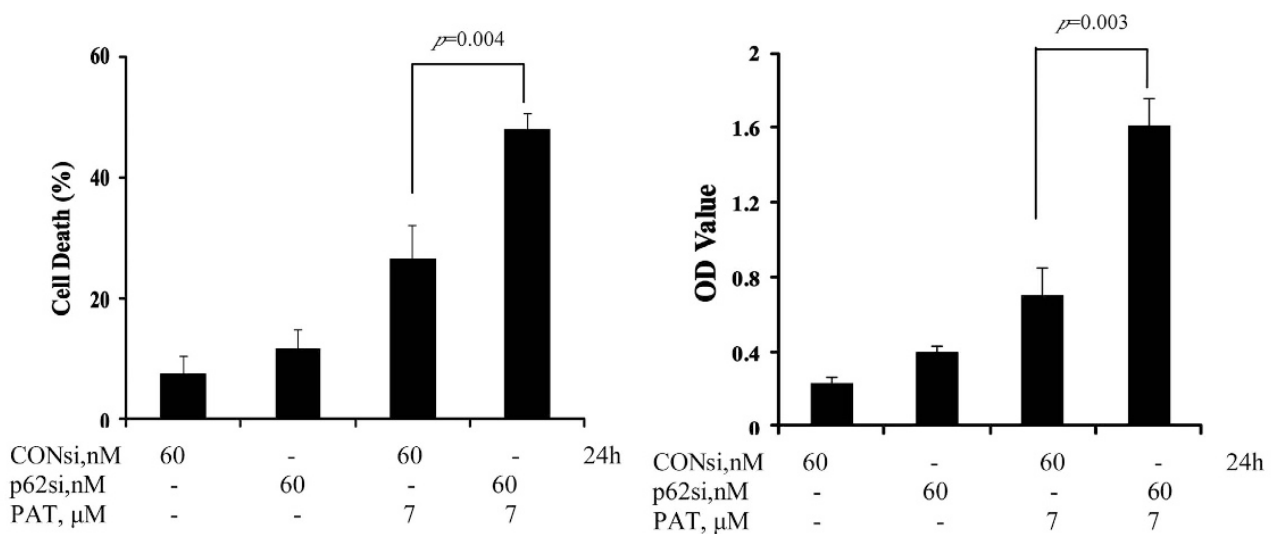

Figure 2 Inhibition of autophagosome degradation-mediated induction of p62 protects PAT-induced apoptosis in HaCaT cells. (a) p62 accumulation induced by PAT in $\mathrm{HaCaT}$ cells. The cells were treated with various concentrations of PAT for $24 \mathrm{~h}$ and then $\mathrm{p} 62$ protein levels were analyzed by western blotting. (b) Effects of autophagosome degradation inhibition by BAF on PAT-induced accumulation of p62. The cells were treated with $7 \mu \mathrm{M}$ PAT for 12 and $24 \mathrm{~h}$ in the presence or absence of $100 \mathrm{nM}$ BAF (added $2 \mathrm{~h}$ before cell harvest) and then $\mathrm{p} 62$ was analyzed by western blotting. (c) Effects of protein synthesis inhibition by CHX on PAT-induced accumulation of p62. The cells were treated with $7 \mu \mathrm{M}$ PAT for $24 \mathrm{~h}$ in the presence or absence of $\mathrm{CHX}$ and then p62 was analyzed by western blotting. Effects of p62 inhibition by RNAi on PAT-induced PARP1 cleavage (d) and apoptosis (e). The cells were transfected with $60 \mathrm{nmol} / \mathrm{l}$ of p62 siRNA using siPORT NeoFX transfection agent. At $24 \mathrm{~h}$ post transfection, the cells were treated with $7 \mu \mathrm{M}$ PAT for $24 \mathrm{~h}$. PARP1 cleavages were analyzed by western blotting and cell death was assessed by Annexin V staining (left) and a Cell Death Detection ELISA kit (right). $n=3,{ }^{\star} P<0.05,{ }^{* \star} P<0.01$, n.S., no significant difference

whether ROS is the downstream signaling of p62 accumulation that mediated ER stress response. We first measured the changes of intercellular ROS levels in response to PAT exposure for the indicated times in $\mathrm{HaCaT}$ cells using flow cytometry following staining with $\mathrm{H}_{2}$ DCFDA, which was considered to specifically detect hydrogen peroxide.
As shown in Figure 5a, PAT caused a rapid increase in ROS levels and a significant change was detected as early as $6 \mathrm{~h}$ exposure. We next examined the role of p62 accumulation in PAT-induced ROS. The cells were transfected with p62 siRNA or non-targeting siRNA. After $24 \mathrm{~h}$ transfection, the cells were treated with PAT for $12 \mathrm{~h}$, and intercellular ROS 


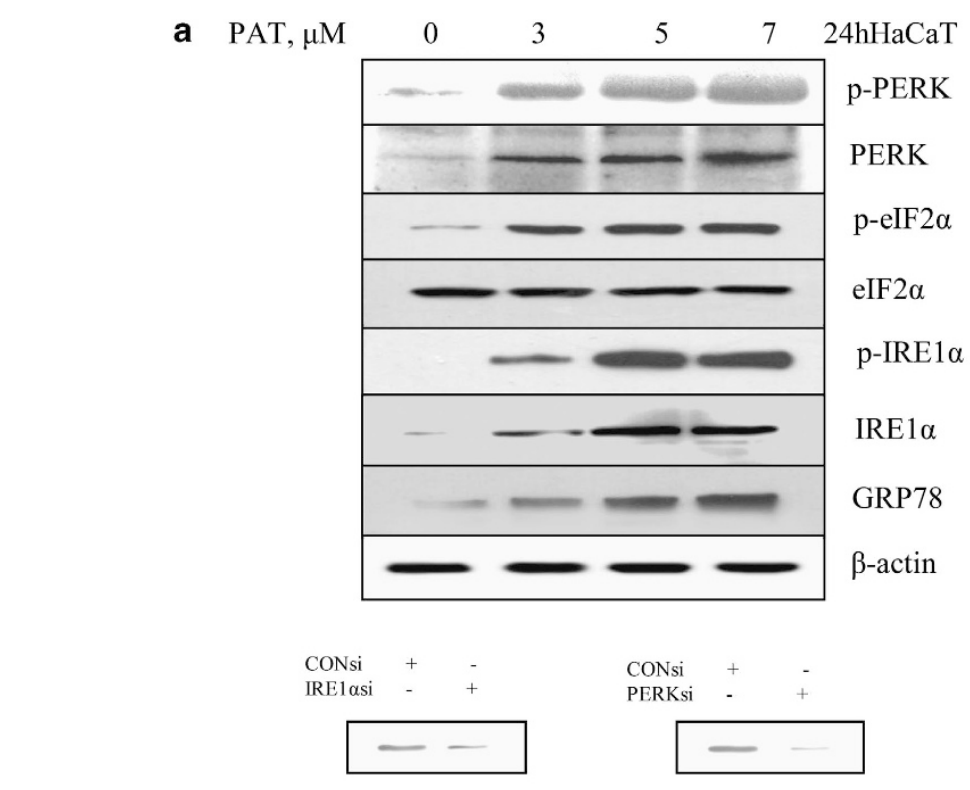

b

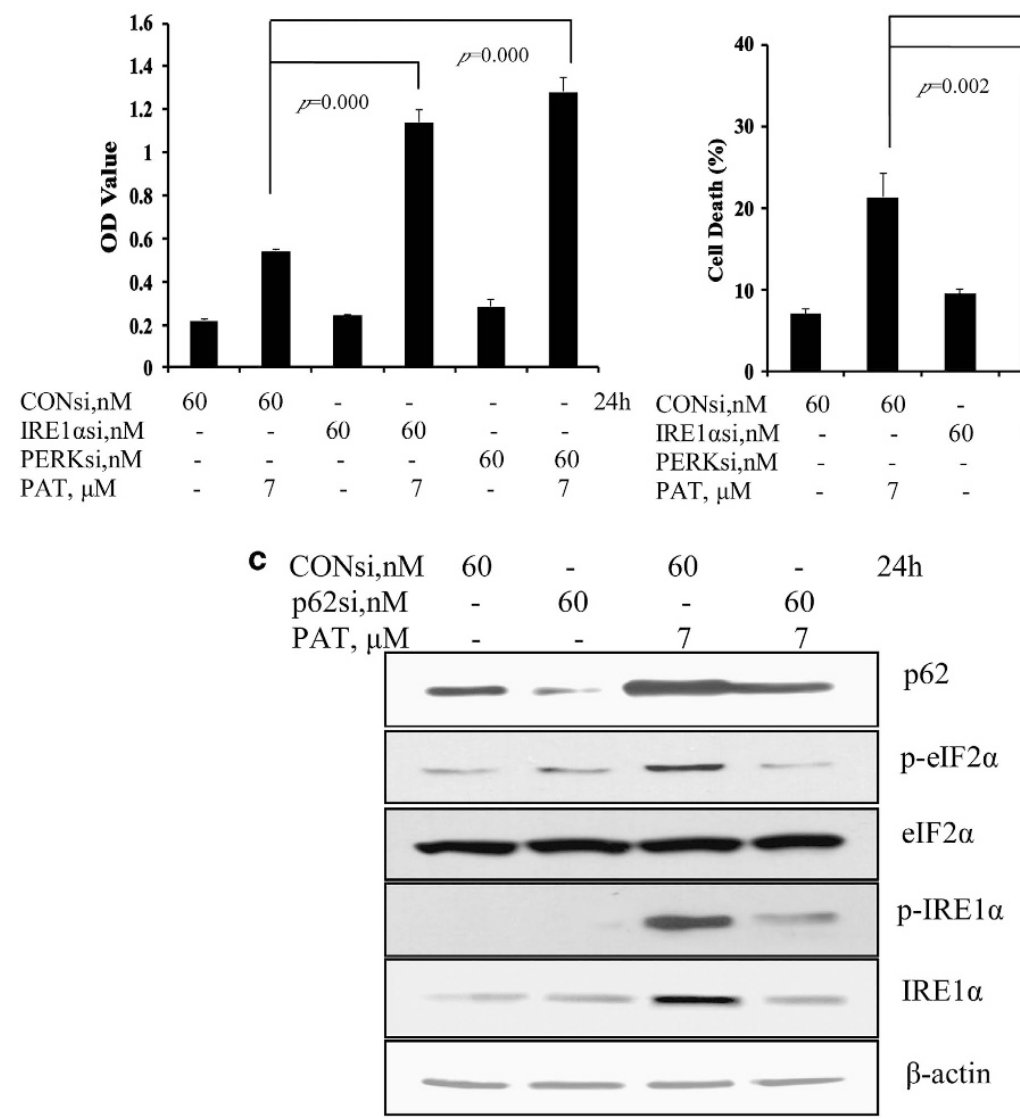

Figure 3 PAT induces p62-mediated cytoprotective ER stress. (a) UPR induced by PAT in HaCaT cells. The cells were treated with various concentrations of PAT for $24 \mathrm{~h}$ and then ER stress associated markers were analyzed by western blotting. (b) Effects of IRE1 $\alpha$ or PERK inactivation by RNAi on PAT-induced apoptosis. The cells were transfected with $60 \mathrm{nmol} / /$ of IRE $1 \alpha$ or PERK siRNA using siPORT NeoFX transfection agent. At $24 \mathrm{~h}$ post transfection, the cells were treated with $7 \mu \mathrm{M}$ PAT for $24 \mathrm{~h}$ and apoptosis induction was assessed by a Cell Death Detection ELISA kit (left) and Annexin V staining (right). (c) Effects of p62 inhibition by RNAi on PAT-induced activation of UPR. The cells were transfected with $60 \mathrm{nmol} / /$ of p 62 siRNA using siPORT NeoFX transfection agent. At $24 \mathrm{~h}$ post transfection, the cells were treated with $7 \mu \mathrm{M}$ PAT for $24 \mathrm{~h}$. IRE $1 \alpha$ and elF2 $\alpha$ phosphorylation were assessed by western blotting

levels were assessed by flow cytometry. As shown in Figure 5b, when p62 was silenced, PAT-induced ROS was nearly abolished, suggesting that p62 induction indeed contributed to the increased ROS generation. To determine the role of ROS in ER stress response induced by PAT, we tested influences of ROS inhibition on the changes of ER 
a $_{\mathrm{B}}$

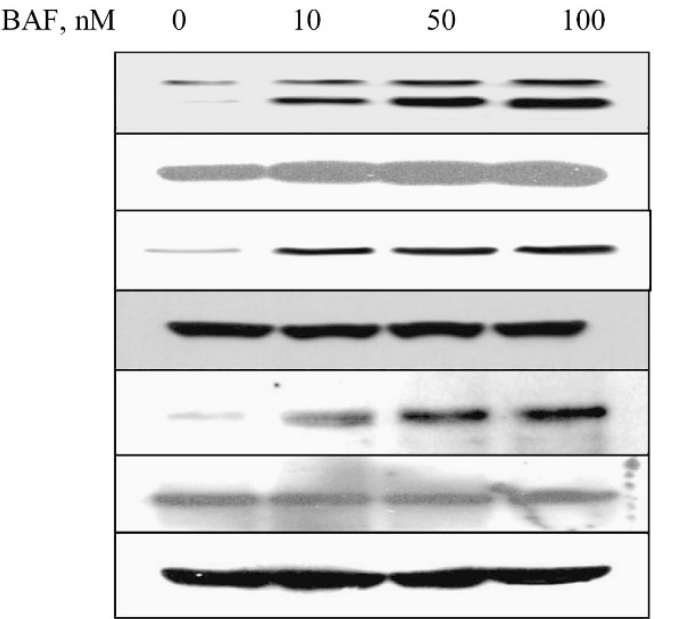

b
CONsi,nM
60
p62si,nM
$\mathrm{BAF}, \mathrm{nM}$
60
60
50
60
50

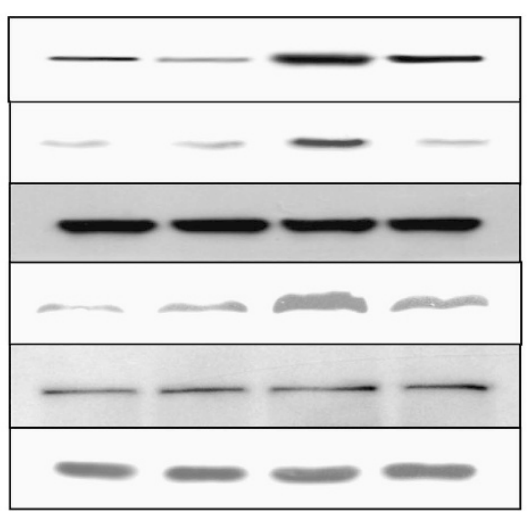

p62

p-eIF $2 \alpha$

eIF $2 \alpha$

p-IRE1 $\alpha$

IRE1 $\alpha$

$\beta$-actin

Figure 4 Inhibition of autophagosome degradation by BAF-induced p62-mediated ER stress. (a) Accumulation of LC3-II/p62 and activation of UPR by $\mathrm{BAF}$ in $\mathrm{HaCaT}$ cells. $\mathrm{HaCaT}$ cells were treated with various concentrations of BAF for $12 \mathrm{~h}$ and then the changes of the key ER stress markers were analyzed by western blotting. (b) Effects of p62 knockdown on BAF-induced activation of UPR. The cells were transfected with $60 \mathrm{nmol} / \mathrm{l}$ of p62 siRNA using siPORT NeoFX transfection agent. At $24 \mathrm{~h}$ post transfection, the cells were treated with BAF for $12 \mathrm{~h}$. IRE $1 \alpha$ and elF2 $\alpha$ phosphorylation were assessed by western blotting

stress markers. The cells were treated with PAT in the presence or absence of $\mathrm{N}$-acetyl-I-cysteine (NAC), ${ }^{21}$ a precursor of intracellular glutathione synthesis and ROS scavenger, for $24 \mathrm{~h}$ and the key ER stress markers were analyzed by western blotting. As shown in Figure 5c, under the condition that ROS was blocked by NAC, PAT-induced phosphorylation of PERK, elF $2 \alpha$ and IRE $1 \alpha$ was nearly abolished, suggesting that ROS had a pivotal role in the p62-mediated ER stress in response to PAT exposure.

p62-mediated cytoprotective ER stress is attributed to ERK1/2-dependent inhibitory BAD phosphorylation. To investigate downstream targets that contributed to p62-mediated cytoprotective ER stress, we examined the changes of the key B-cell CLL/lymphoma 2 (BCL2) family proteins in response to PAT. As shown in Figure 6a, PAT induced a slight decrease of BCL2, B-cell lymphoma a

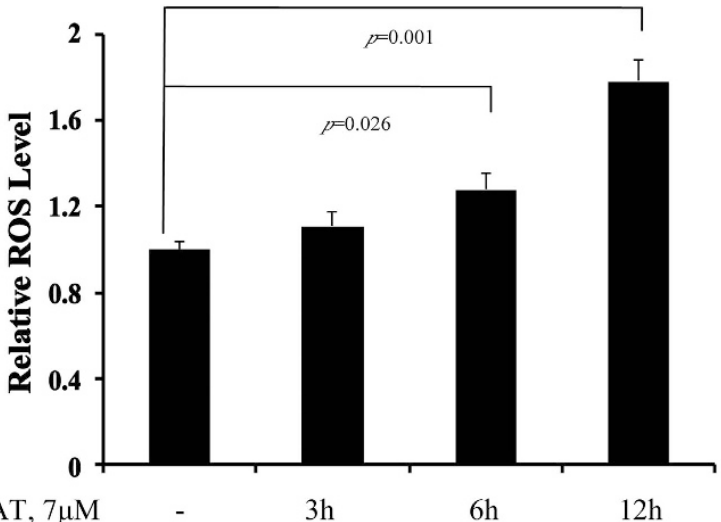

PAT, $7 \mu \mathrm{M}$

$12 \mathrm{~h}$

b

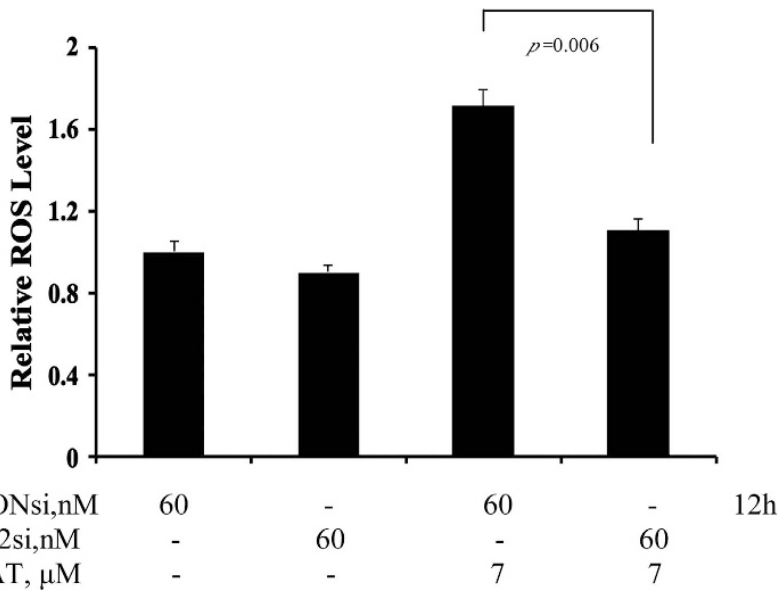

$\begin{array}{llccccc}\text { C } & \mathrm{NAC}, \mathrm{mM} & - & 10 & - & 10 & 24 \mathrm{~h} \\ \mathrm{PAT}, \mu \mathrm{M} & - & - & 7 & 7 & \end{array}$

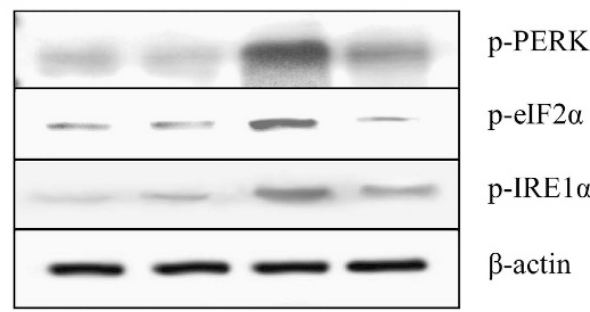

Figure 5 ROS is involved in PAT-induced p62-mediated ER stress. (a) PAT induced ROS generation in a time-dependent manner in $\mathrm{HaCaT}$ cells. The cells were treated with PAT for the indicated times and intercellular ROS levels in response to PAT exposure were measured by flow cytometry following staining with $\mathrm{H}_{2} \mathrm{DCFDA}$. (b) Influences of p62 knockdown on ROS generation by PAT. (c) Effects of ROS inhibition by NAC on UPR activation by PAT. The cells were treated with PAT in the presence or absence of NAC for $24 \mathrm{~h}$ and then the key ER stress markers were analyzed by western blotting

extra-large (Bcl-xL) and myeloid cell leukemia sequence 1 (MCL1) expression, whereas a significant increase of BAD inhibitory phosphorylation (ser-112) by PAT was detected. Given BAD phosphorylation on ser-112 is an important residue targeted by $E R K 1 / 2$ kinase, ${ }^{22}$ we further measured the changes of ERK1/2 phosphorylation. As shown in Figure 6a, PAT treatment caused an increased phosphorylation of ERK1/2, which correlated very closely with BAD ser-112 phosphorylation. To determine whether the increased phosphorylation of ERK1/2-BAD was attributed to ER stress 
a

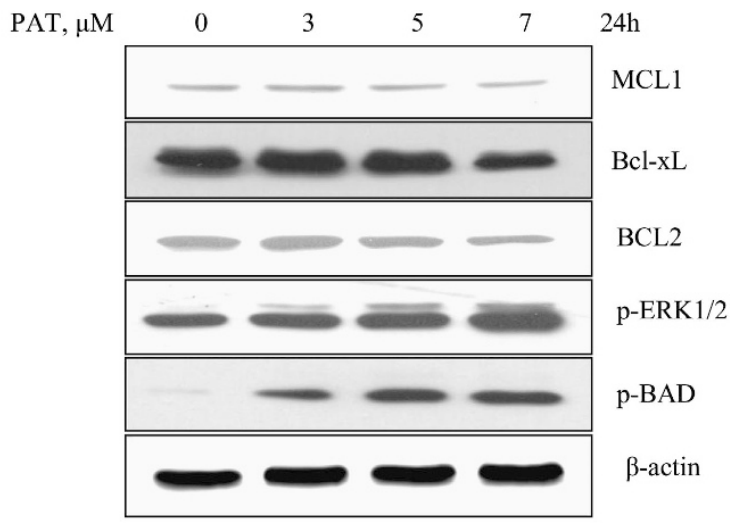

b

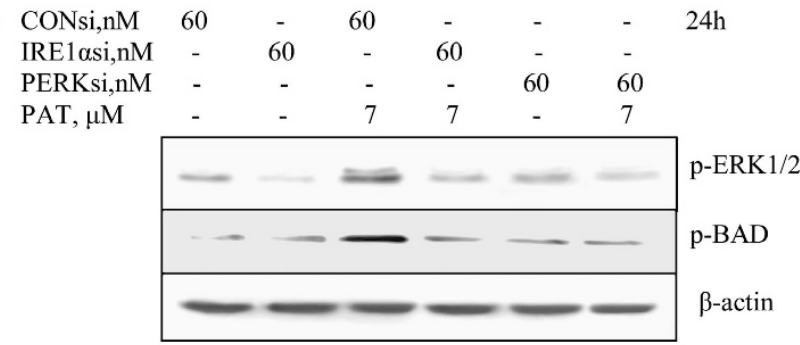

C

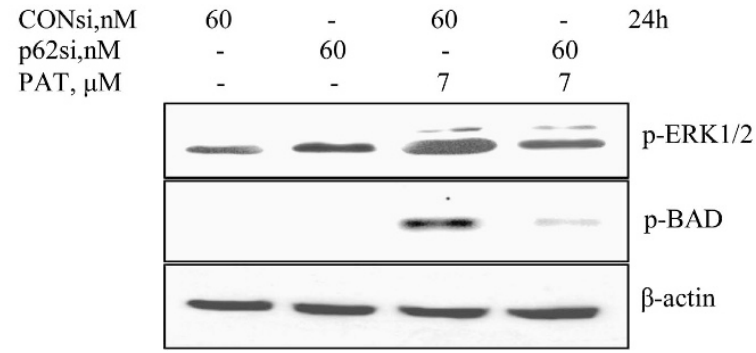

Figure 6 p62-mediated cytoprotective ER stress is attributed to ERK1/2dependent inhibitory BAD phosphorylation. (a) Regulation of PAT on BCL2 family proteins and ERK1/2. The cells were treated with various concentrations of PAT for $24 \mathrm{~h}$ and then the expression of BCL2, Bcl-xL, MCL1 and phosphorylation of ERK1/2 and BAD were analyzed by western blotting. (b) Effects of UPR inactivation by RNAi on PAT-induced phosphorylation of ERK1/2 and BAD. (c) Influences of p62 inhibition on PAT-induced phosphorylation of ERK1/2 and BAD

response, we tested effects of IRE $1 \alpha$ or PERK knockdown on PAT-induced phosphorylation of ERK $1 / 2$ and BAD. As shown in Figure $6 b$, under the condition that IRE $1 \alpha$ or PERK was silenced, PAT-induced ERK1/2 and BAD phosphorylation were abolished. Consistent with the above results, inhibition of p62 by RNAi led to a significant decrease of ERK1/2 and BAD phosphorylation induced by PAT. Taken together, these results suggest that ERK1/2-mediated inactivation of BAD might contribute to p62-mediated cytoprotective ER stress in response to PAT exposure.

Autophagy inhibition by PAT is associated with its inhibitory effect on CTSB and CTSD. It has been shown that PAT has a strong affinity for sulfhydryl groups. ${ }^{23}$ Given the importance of thiol groups for the activities of lysosomal enzymes, we then asked whether autophagy inhibition by PAT was attributed to suppression of lysosomal enzymes. The cleaved (active) forms of the two key lysosomal proteases, CTSB and CTSD were analyzed by western

$\begin{array}{lllllll}\text { a } \mathrm{PAT}, 7 \mu \mathrm{M} & 0 & 3 & 6 & 12 & 24 & \text { (h) }\end{array}$

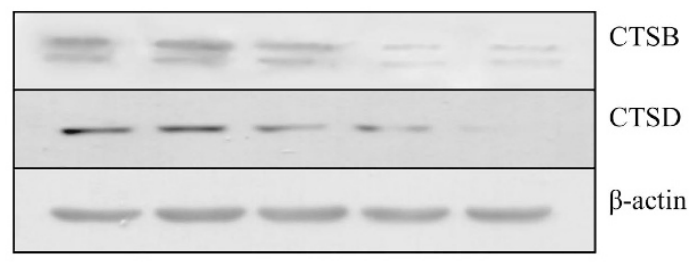

b

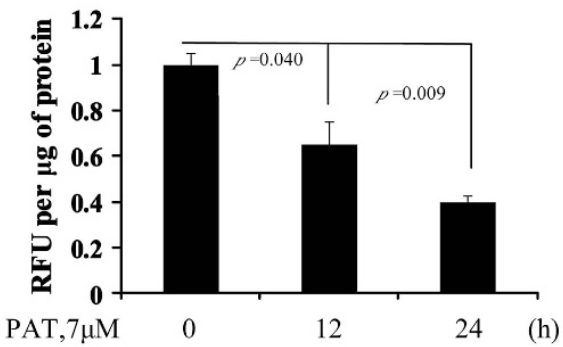

C

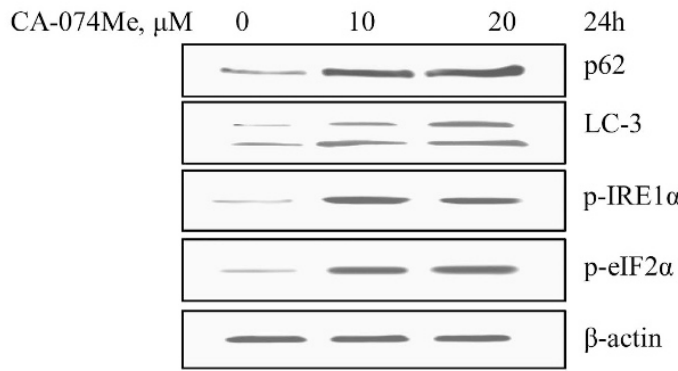

Figure 7 Autophagy inhibition by PAT is associated with its inhibitory effect on CTSB and CTSD. (a) Inhibitory effects of PAT on activity of lysosomal enzymes CTSB and CTSD measured by western blotting. The cells were treated with PAT for the indicated times and then the cleaved forms of CTSB and CTSD were examined by western blotting. (b) Effects of PAT on CTSB activity measured by CTSB activity assay kit $(n=3)$. (c) Inhibition of CTSB was sufficient to induce accumulation of LC3-II/p62 and activation of UPR. The cells were treated with CTSB inhibitor CA-074Me for $24 \mathrm{~h}$ and then LC3-I to LC3-II conversion, p62 and the key ER stress markers were assessed by western blotting

blotting. As shown in Figure 7a, treatment with PAT caused a time-dependent decrease of cleavages of CTSB and CTSD. Consistently, enzyme activity assay revealed that CTSB activity was considerably reduced in PAT-treated cells (Figure $7 \mathrm{~b}$ ). To determine the role of the reduction of Iysosomal enzyme activity in autophagy inhibition and UPR activation, we tested effects of CA-074Me, a specific CTSB inhibitor, ${ }^{24}$ on LC3-II and p62 accumulation and changes of the key ER stress markers assessed by western blotting. As shown in Figure 7c, treatment with CA-074Me led to a significant increase in LC3-II/p62 accumulation and IRE1 $\alpha /$ elF2 $\alpha$ phosphorylation, suggesting that inhibition of CTSB is sufficient for autophagy inhibition and ER stress response.

\section{Discussion}

The skin is a probable toxicological target of PAT. Previous study has shown that PAT has potential skin carcinogenicity. ${ }^{13}$ In the current study, we demonstrated that treatment with PAT resulted in autophagy inhibition, which in turn activated p62-mediated pro-survival signaling in human keratinocyte $\mathrm{HaCaT}$ cells. Given the important role of 
autophagy in carcinogenesis, our data suggested that inhibition of autophagy might contribute to PAT-induced skin carcinogenesis. These findings provided a novel mechanistic support for PAT as a potential skin carcinogen.

The implication of $p 62$ in carcinogenesis still remains controversial. Accumulation of p62 functions either as prooncogenic or anti-oncogenic signaling. ${ }^{25}$ Our current study showed that PAT induced autophagy inhibition-mediated p62 accumulation and inhibition of $p 62$ by RNAi resulted in an increase of apoptosis, suggesting p62 is a pro-survival signaling, which provided survival advantage of the cells and might resulted in disruption of the balance between cell dying and growing, which was thought to be an early and important event in carcinogenic process. In terms of mechanisms involved in pro-survival function of p62, our data revealed that p62 accumulation caused a cytoprotective ER stress. Moreover, we identified that PAT-induced cytoprotective ER stress was associated with ERK1/2-mediated BAD phosphorylation. Our findings for the first time demonstrated that the pro-survival function of p62 was attributed to the inhibitory phosphorylation of BAD via IRE $1 \alpha /$ PERK-mediated ERK1/2 activation. It has been well documented that ROS contributed to the induction of genomic instability and carcinogenesis. ${ }^{26}$ Our data showed that an increased ROS generation was detected in response to PAT exposure, which was attributed to p62 accumulation. In line with our current in vitro findings, an enhanced ROS generation and activation of ERK1/2 have been found in vivo in PAT-treated mouse skin. ${ }^{13}$ Together, our findings suggest that PAT-induced p62 accumulation might exert pro-oncogenic function through ROS generation and disruption of apoptosis.

It has been shown that autophagy inhibition can induce ER stress. ${ }^{10,19}$ However, the mechanisms underlying autophagy inhibition-induced ER stress have not been well elucidated. In the present study, we found that inhibition of autophagy by PAT resulted in the activation of ER stress responses. Inhibition of $\mathrm{p} 62$ by RNAi led to abolishment of ER stress responses induced by PAT. Furthermore, this finding was further verified in a specific autophagy inhibitor BAF-induced autophagy inhibition, suggesting that p62 had a critical role in autophagy inhibition-mediated ER stress. Regarding the mechanism by which p62 activated ER stress responses, our data demonstrated that blockade of ROS by NAC prevented PAT-induced elF2 $\alpha$ and IRE $1 \alpha$ phosphorylation, whereas p62 silencing suppressed ROS generation, indicating that ROS is a possible mediator of PAT-induced p62-dependent ER stress. Our findings provided a novel mechanistic support for ER stress induced by autophagy inhibition.

Our previous study showed that autophagy induction by ursolic acid induced ER stress, ${ }^{27}$ whereas the current findings demonstrated that inhibition of basal level autophagy triggered activation of UPR. These data indicate that autophagy can bidirectionally regulate ER stress that may depend on the levels of autophagy. Basal level of autophagy functions as suppressor of ER stress, whereas excess of autophagy becomes activator of ER stress. The mechanisms for this paradoxical effect of autophagy on ER stress are being investigated.

The expression of p62 is regulated at both the transcriptional and post-translational levels. ${ }^{28,29}$ Autophagy-dependent mechanism has a critical role in the control of intracellular levels of p62. ${ }^{30,31}$ Our data showed that treatment with PAT caused an increased autophagosome levels, accompanied by p62 accumulation. Under the condition that autophagosome degradation is blocked by its inhibitor BAF, LC3-II levels were not further increased by PAT treatment, suggesting that the inhibition of autophagosome degradation is a reasonable explanation for PAT-induced p62 accumulation. This notion was further confirmed by the evidence that PAT still can increase p62 protein levels when the novel protein synthesis was blocked by $\mathrm{CHX}$. Taken together, our data indicated that inhibition of autophagy was at least one mechanism involved in PAT-induced p62 accumulation.

In summary (Figure 8), PAT, a moldy fruit mycotoxin, inhibited basal autophagy and induced p62 accumulation in human keratinocyte HaCaT cells. Accumulation of p62 led to an increased ROS generation and UPR-ERK1/2-mediated BAD inhibitory phosphorylation (disruption of apoptosis). Our findings suggest that PAT might exert oncogenic activity via its ability to suppress autophagy and to induce p62mediated pro-survival signaling.

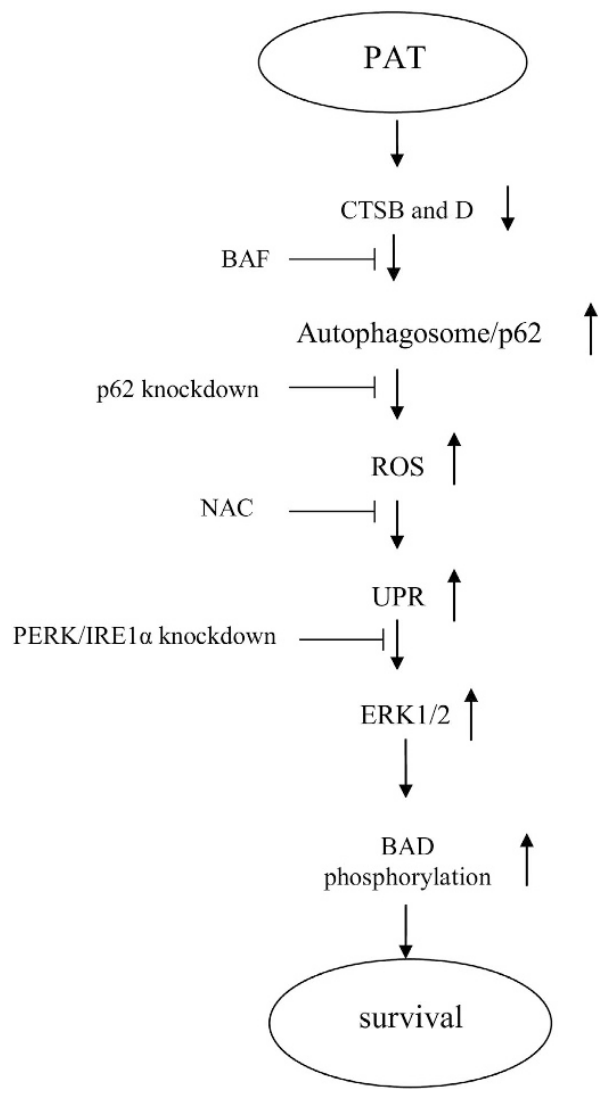

Figure 8 Signaling pathways underlying PAT-induced p62-mediated pro-survival effects in HaCaT cells. PAT exposure led to a reduction of lysosomal enzyme CTSB and CTSD activity, which in turn caused autophagosome degradation inhibition and p62 accumulation. The accumulation of $p 62$ resulted in ROS generation, followed by cytoprotective ER stress response. The cytoprotective effects of p62-mediated ER stress were associated with ERK1/2-dependent BAD inhibitory phosphorylation 


\section{Materials and Methods}

Chemicals and reagents. PAT (P1639), 3-MA (M9281), BAF (B1793), NAC (A7250), CA-074Me (C5857), CHX (01810), $\mathrm{H}_{2}$ DCFDA (D6883) were purchased from Sigma-Aldrich (St. Louis, MO, USA). Antibodies specific for GRP78 (3183S), phospho-ERK (4094S), phospho-BAD (Ser-112) (5284S), MCL1 (4572S), BCL2 (2870S), BCL-XL (2764S), p-elF2 $\alpha$ (3398S), $\beta$-actin (4970) and cleaved PARP1 p89 (9548S) were purchased from Cell Signaling Technology (Denvers, MA, USA). Antibody for phospho-PERK (SC-35277) was purchased from Santa Cruz Biotechnology (Santa Cruz, CA, USA). Antibody for phospho-IRE1 $\alpha$ (ab124945) was purchased from Abcam (Cambridge, UK). Antibody for LC3 (PM036), p62 (M162-3) and ATG5 (M153-3) was purchased from MBL International corporation (Woburn, MA, USA).

Cell culture and treatments. HaCaT and 293T cell lines were grown in Dulbecco's Modification of Eagle's Medium (DMEM) (Thermo, Waltham, MA, USA; SH30022.01B) supplemented with $10 \%$ fetal bovine serum without antibiotics. At 24-48 h after plating when cells were $50-60 \%$ confluence, the medium was changed before starting the treatment with PAT and/or other agents.

Apoptosis evaluation. Apoptosis was assessed by two methods. The first one was Annexin V staining of externalized phosphatidylserine in apoptotic cells by flow cytometry using Annexin V/FITC staining kit from MBL International. The second method was a Cell Death ELISA kit to detect cytoplasmic histone-associated DNA fragments purchased from Roche Diagnostics (Indianapolis, IN, USA).

Autophagy detection. Autophagy induction was determined by two methods. The first method was western blotting for conversion of the LC3-I to LC3-II. The second one was immunofluorescence staining for LC3 localization as reported previously. ${ }^{16,32}$

Measurement of ROS. Generation of intercellular ROS was measured by flow cytometry following staining with $\mathrm{H}_{2}$ DCFDA. ${ }^{33} \mathrm{H}_{2}$ DCFDA is a reduced form of 2,7-dichlorofluorescein. Oxidation by hydrogen peroxide can be detected by monitoring the increase in green fluorescence with a flow cytometer. At $30 \mathrm{~min}$ before harvest, $\mathrm{H}_{2} \mathrm{DCFDA}$ was added to the medium to a concentration of $5 \mu \mathrm{mol} / \mathrm{l}$. The fluorescence was measured using a Becton Dickinson flow cytometer.

Western blotting. The cell lysate was prepared in ice-cold radioimmunoprecipitation assay buffer. Cell lysates were resolved by electrophoresis and transferred to a polyvinylidene fluoride (PVDF) membrane (Millipore, Billerica, MA, USA; IPVH00010). The blot was then probed with primary antibody followed by incubation with the appropriate horseradish peroxidase-conjugated secondary antibodies (MBL, 330 and 458). The signal was visualized by enhanced chemiluminescence (Fisher/Pierce, Rockford, IL, USA; 32106) and recorded on an X-ray film (Eastman Kodak Company, Rochester, NY, USA; XBT-1).

RNA interference. siRNAs targeting PERK (sc-36213), IRE1 $\alpha$ (sc-40705) were purchased from Santa Cruz Biotechnologies. siRNAs targeting ATG5, p62 and non-targeting siRNA were synthesized by Integrated DNA Technologies (Coralville, IA, USA). The cells were transfected with $60 \mathrm{nmol} / \mathrm{I}$ of specific or non-targeting siRNA using siPORT NeoFX transfection agent (AM4510) for $24 \mathrm{~h}$ and then were used for subsequent experiments.

CTSB activity assay. The CTSB activity in the samples was detected in reaction buffer containing substrate sequence RR labeled with AFC (amino-4trifluoromethyl coumarin) according to the manufacturer's instructions (BioVision, Mountain View, CA, USA; K140-100). After $2 \mathrm{~h}$ of incubation at $37^{\circ} \mathrm{C}$ in the dark, the samples were analyzed with Ex400 nm-Em $505 \mathrm{~nm}$. All fluorescence readings are expressed in relative fluorescence units (RFU), CTSB activity was expressed as the RFU per $\mu \mathrm{g}$ of protein sample.

Statistical analysis. Data are presented as mean \pm S.D. These data were analyzed with the ANOVA with appropriate post hoc comparison among means. $P<0.05$ was considered statistically significant.

\section{Conflict of Interest}

The authors declare no conflict of interest.
Acknowledgements. This work was supported by grants from National Natural Science Foundation of China (NSFC, 31371752, 31071533, 30972172) and Ministry of Science and Technology of China (2012BAD33B09).

1. Wang K, Klionsky DJ. Mitochondria removal by autophagy. Autophagy $2011 ; 7: 297-300$.

2. Qu X, Yu J, Bhagat G, Furuya N, Hibshoosh $\mathrm{H}$, Troxel A et al. Promotion of tumorigenesis by heterozygous disruption of the beclin 1 autophagy gene. J Clin Invest 2003; 112: 1809-1820.

3. Yue Z, Jin S, Yang C, Levine AJ, Heintz N. Beclin 1, an autophagy gene essential for early embryonic development, is a haploinsufficient tumor suppressor. Proc Natl Acad Sci USA. 2003; 100: 15077-15082.

4. Levine B, Kroemer G. Autophagy in the pathogenesis of disease. Cell 2008; 132: 27-42.

5. Takamura A, Komatsu M, Hara T, Sakamoto A, Kishi C, Waguri S et al. Autophagydeficient mice develop multiple liver tumors. Genes Dev 2011; 25: 795-800.

6. Inami Y, Waguri S, Sakamoto A, Kouno T, Nakada K, Hino O et al. Persistent activation of Nrf2 through p62 in hepatocellular carcinoma cells. J Cell Biol 2011; 193: 275-284.

7. Hart LS, Cunningham JT, Datta T, Dey S, Tameire F, Lehman SL et al. ER stress-mediated autophagy promotes Myc-dependent transformation and tumor growth. J Clin Invest 2012; 122: 4621-4634.

8. Degenhardt K, Mathew R, Beaudoin B, Bray K, Anderson D, Chen G et al. Autophagy promotes tumor cell survival and restricts necrosis, inflammation, and tumorigenesis. Cancer Cell 2006; 10: 51-64.

9. Puissant A, Fenouille N, Auberger P. When autophagy meets cancer through p62/SQSTM1. Am J Cancer Res 2012; 2: 397-413.

10. Mathew R, Karp CM, Beaudoin B, Vuong N, Chen G, Chen HY et al. Autophagy suppresses tumorigenesis through elimination of p62. Cell 2009; 137: 1062-1075.

11. Kwon O, Soung NK, Thimmegowda NR, Jeong SJ, Jang JH, Moon DO et al. Patulin induces colorectal cancer cells apoptosis through EGR-1 dependent ATF3 up-regulation. Cell Signal 2012; 24: 943-950.

12. Puel Olivier, Galtier Pierre, Oswald Isabelle P. Biosynthesis and toxicological effects of patulin. Toxins 2010; 2: 613-631.

13. Saxena N, Ansari KM, Kumar R, Chaudhari BP, Dwivedi PD, Das M. Role of mitogen activated protein kinases in skin tumorigenicity of patulin. Toxicol Appl Pharmacol 2011; 257: 264-271.

14. Wu YT, Tan HL, Shui G, Bauvy C, Huang Q, Wenk MR et al. Dual role of 3-methyladenine in modulation of autophagy via different temporal patterns of inhibition on class I and III phosphoinositide 3-kinase. J Biol Chem 2010; 285: 10850-10856.

15. Yoshimori T, Yamamoto A, Moriyama Y, Futai M, Tashiro Y. Bafilomycin A1, a specific inhibitor of vacuolar-type $\mathrm{H}+$-ATPase, inhibits acidification and protein degradation in Iysosomes of cultured cells. J Biol Chem 1991; 266: 17707-17712.

16. Kimura S, Noda T, Yoshimori T. Dissection of the autophagosome maturation process by a novel reporter protein, tandem fluorescent-tagged LC3. Autophagy 2007; 3: 452-460.

17. Zhang YB, Gong JL, Xing TY, Zheng SP, Ding W. Autophagy protein p62/SQSTM1 is involved in HAMLET-induced cell death by modulating apoptosis in U87MG cells. Cell Death Dis 2013; 4: e550.

18. Komatsu M, Kageyama S, Ichimura Y. p62/SQSTM1/A170: physiology and pathology. Pharmacol Res 2012; 66: 457-462.

19. Xu Y, Yu H, Qin H, Kang J, Yu C, Zhong J et al. Inhibition of autophagy enhances cisplatin cytotoxicity through endoplasmic reticulum stress in human cervical cancer cells. Cancer Lett 2012; 314: 232-243.

20. Linares JF, Amanchy R, Greis K, Diaz-Meco MT, Moscat J. Phosphorylation of 62 by cdk1 controls the timely transit of cells through mitosis and tumor cell proliferation. $\mathrm{Mol}$ Cell Biol 2011; 31: 105-117.

21. Ritchie IR, Dyck DJ. Rapid loss of adiponectin-stimulated fatty acid oxidation in skeletal muscle of rats fed a high fat diet is not due to altered muscle redox state. PLoS One 2012; 7: e52193.

22. Bonni A, Brunet A, West AE, Datta SR, Takasu MA, Greenberg ME. Cell survival promoted by the Ras-MAPK signaling pathway by transcription-dependent and -independent mechanisms. Science 1999; 286: 1358-1362.

23. Glaser N, Stopper H. Patulin: mechanism of genotoxicity. Food Chem Toxicol. 2012; 50: 1796-1801.

24. Matarrese P, Ascione B, Ciarlo L, Vona R, Leonetti C, Scarsella M et al. Cathepsin B inhibition interferes with metastatic potential of human melanoma: an in vitro and in vivo study. Mol Cancer 2010; 9: 207.

25. Moscat J, Diaz-Meco MT. p62: a versatile multitasker takes on cancer. Trends Biochem Sci 2012; 37: 230-236.

26. Ziech D, Franco R, Pappa A, Panayiotidis Ml. Reactive oxygen species (ROS)-induced genetic and epigenetic alterations in human carcinogenesis. Mutat Res 2011; 711: 167-173.

27. Zhao C, Yin S, Dong Y, Guo X, Fan L, Ye M et al. Autophagy-dependent PERK activation compromises ursolic acid-induced apoptosis through upregulation of MCL1 in MCF-7 human breast cancer cells. Autophagy 2013; 9: 196-207.

28. Duran A, Linares JF, Galvez AS, Wikenheiser K, Flores JM, Diaz-Meco MT et al. The signaling adaptor p62 is an important NF-kappaB mediator in tumorigenesis. Cancer Cell 2008; 13: 343-354. 
29. Ling J, Kang Y, Zhao R, Xia Q, Lee DF, Chang Z et al. KrasG12D-induced IKK2/beta/ NF-kappaB activation by IL-1alpha and p62 feedforward loops is required for development of pancreatic ductal adenocarcinoma. Cancer Cell 2012; 21: 105-120.

30. Klionsky DJ, Abeliovich H, Agostinis P, Agrawal DK, Aliev G, Askew DS et al. Guidelines for the use and interpretation of assays for monitoring autophagy in higher eukaryotes Autophagy 2008; 8: 445-544.

31. Moscat Jorge, Diaz-Meco Maria T. p62 at the crossroads of autophagy, apoptosis, and cancer. Cell 2009; 137: 1001-1004.

32. Herman-Antosiewicz A, Johnson DE, Singh SV. Sulforaphane causes autophagy to inhibit release of cytochrome $\mathrm{C}$ and apoptosis in human prostate cancer cells. Cancer Res 2006; 66: $5828-5835$.
33. Rothe G, Valet G. Flow cytometric analysis of respiratory burst activity in phagocytes with hydroethidine and 2'7'-dichlorofluorescein. J Leuk Biol 1990; 47: 440-448.

(c) (5) (2) Cell Death and Disease is an open-access journal published by Nature Publishing Group. This work is licensed under a Creative Commons Attribution-NonCommercialShareAlike 3.0 Unported License. To view a copy of this license, visit http://creativecommons.org/licenses/by-nc-sa/3.0/

Supplementary Information accompanies this paper on Cell Death and Disease website (http://www.nature.com/cddis) 